\title{
Hepatic endometriosis: a rare case and review of the literature
}

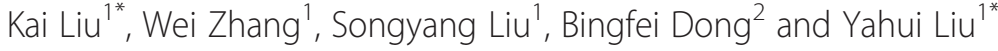

\begin{abstract}
Hepatic endometriosis is one of the rarest disorders characterized by the presence of ectopic endometrium in the liver. To our knowledge, only 21 cases of hepatic endometrioma have been described in the medical literature. We report a case of a 36-year-old primiparous female with hepatic endometriosis forming a large cystic mass. The patient presented once with severe right quadrant pain as her only symptom and no history of endometriosis. Complete blood count and biochemical tests were normal. Abdominal ultrasonography and computed tomography scans suggested the presence of a $6.5 \times 6.0 \mathrm{~cm}$ cystic mass in segment III of the liver. The mass was completely removed by local liver resection. The intraoperative frozen sections suggested a diagnosis of hepatic endometriosis. The diagnosis was confirmed through histological immunostaining without intrinsic abnormality. A preoperative diagnosis of hepatic endometriosis is made on the basis of considering the possibility in advance. Hepatic endometriosis should be considered in the differential diagnosis of a cystic liver mass despite conducting exhaustive investigations in the absence of characteristic clinical and radiological features. Histological examination is essential, and surgery remains the treatment of choice.
\end{abstract}

Keywords: Hepatic endometriosis, Hepatic cysts, Atypical endometriosis, Differential diagnosis

\section{Background}

Endometriosis is a benign condition most commonly noted in the uterus, fallopian tubes, ovaries, and local pelvic peritoneum [1]. It affects approximately $10 \%$ of women of reproductive age and $2.5 \%$ of postmenopausal women [2]. However, endometriotic lesions have also been described in almost all other remote organs of the human body, including the omentum, gastrointestinal tract, peritoneum, operative scars, lymph nodes, umbilicus, skin, lungs, pleura, bladder, kidneys, pancreas, and even in males [3]. Hepatic endometriosis, one of the rarest forms of atypical endometriosis, was first described in 1986 [4]. To our knowledge, only 21 cases of hepatic endometriosis have been previously reported in the literature. We herein describe the 22nd case of hepatic endometriosis and evaluate the current literature addressing the diagnosis of hepatic endometriosis focusing on advances in the clinical manifestation, pathogenesis, and diagnostic workup.

\footnotetext{
*Correspondence: 6kai@163.com; liuyahui588@126.com

'Department of Hepatobiliary and Pancreatic Surgery, No.71 Xinmin Street,

First Hospital, Jilin University, Changchun 130021, China

Full list of author information is available at the end of the article
}

\section{Case presentation}

A 36-year-old woman, primigravida, consulted our hospital for investigation of a cystic hepatic lesion in August 2013. The patient had no history of endometriosis. Half a year previously, the patient had presented with severe right quadrant pain in the midnight which only lasted for $3 \mathrm{~h}$ just before menstruation. An upper abdominal ultrasound showed a $6-\mathrm{cm}$ lesion within the left lobe of the liver. The patient was diagnosed with a hepatic cystic mass in segment III of the left lobe. A laparotomy had been considered and postponed since the patient had managed to cope with her symptoms. The patient refused further treatment at that moment. The patient was not currently undergoing menopause. The patient had no exposure to hepatotoxic drugs, estrogens, progestins, or oral contraceptives. There were no other significant symptoms. On physical examination, no definite abdominal mass lesion was palpable and no lymphadenopathy was noted. Complete blood count was within normal range as were the liver function tests. Serological tests for hepatitis B surface antigen and anti-hepatitis $C$ virus antibodies were negative. The tumor markers (carcinoembryonic antigen, carbohydrate antigen 19-9, alpha- 
fetal protein, carbohydrate antigen 125) were normal, as was the routine lab work. A preoperative computed tomography scan showed a well-circumscribed cystic lesion of $6.5 \times 6.0 \mathrm{~cm}$ located in segment III (Figure 1). The wall appeared thick with complex septate. A preoperative tentative diagnosis included liver cystadenoma or liver cystadenocarcinoma.

An exploratory operation was performed, which revealed a large cystic tumor occupying segment III of the liver (Figure 2). There was no evidence of metastases. Biopsies were taken from the cyst wall, and the intraoperative frozen section histology suggested a diagnosis of hepatic endometriosis. The endometriosis was completely removed by a pericystectomy. An abdominal cavity exploration revealed no other pathologic events. The pelvis was examined and no evidence of endometriosis was found. We did not detect any other abnormalities during the operation. The patient made an uneventful recovery and was discharged on the ninth postoperative day. After 3 months of follow-up, the patient is asymptomatic with no evidence of recurrent disease.

Histopathology analysis revealed a lobulated cyst with adjacent normal hepatic parenchyma. The cyst wall was partially composed of endometrial glandular and stromal elements (Figure 3a), characteristic of endometriosis. Immunostaining of the stromal cell and epithelial cells expressed strong coloring for estrogen and progesterone receptor (Figure $3 \mathrm{~b}, \mathrm{c}$ ). This was further confirmed by positive immunostaining of CD10, CK7, as well as HepPar-1, which proved the epithelial origin of the cyst (Figure 3d,e, f). Since no atypical cells were detected, we concluded the diagnosis to be benign intrahepatic endometriosis.

\section{Discussion}

Endometriosis is defined as the presence of endometriallike tissue outside the uterus, which induces a chronic,

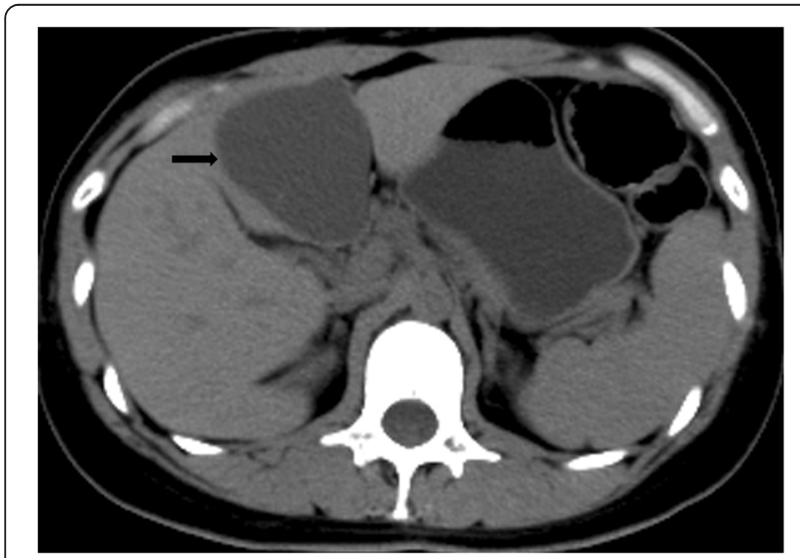

Figure 1 Hepatic CT scan showing a well circumscribed cystic lesion of $6.5 \times 6.0 \mathrm{~cm}$ located in segments III of the left liver lobe.

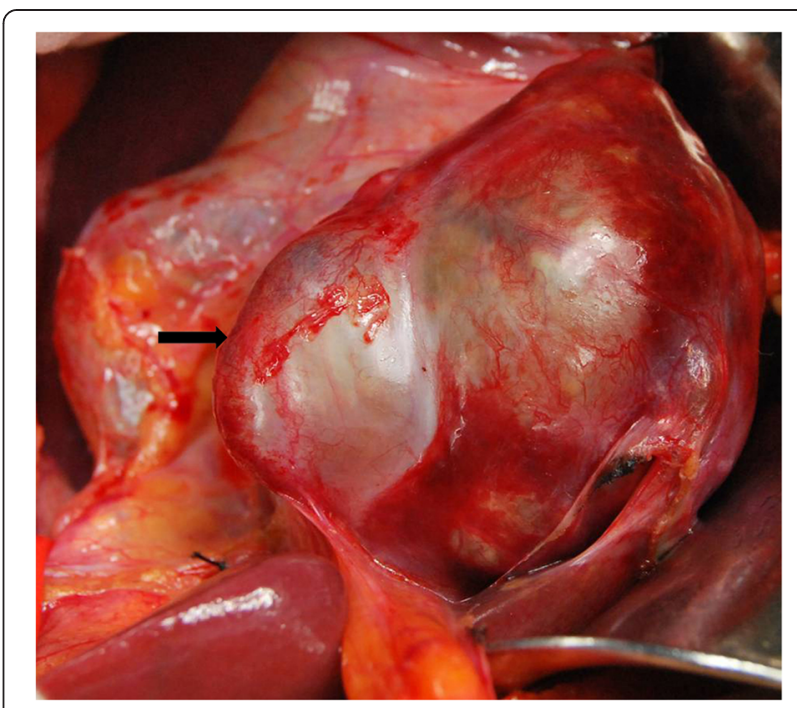

Figure 2 Macroscopic examination of the liver mass. A $6.5 \times$ $6.0 \times 5.5 \mathrm{~cm}$ cystic mass was presented in the left lobe of the liver with thick fibrous capsule.

inflammatory reaction [1]. It affects approximately $10 \%$ of women of reproductive age and $2.5 \%$ of postmenopausal women, from all ethnic and social groups [2]. Although clinical and imaging features of typical endometriosis within the pelvis can be highly suggestive, the gold standard for diagnosis is based on the histologic examination of appropriately sampled tissue [5]. Endometriosis was first described by Rokitansky in 1860 . The most commonly affected sites are the pelvic organs (ovaries, fallopian tubes, uterosacral ligaments, Pouch of Douglas) and peritoneum, although other parts of the body such as the lungs are occasionally affected. However, endometriotic lesions have also been described in some other unusual remote organs of the human body, including the omentum, gastrointestinal tract, operative scars, lymph nodes, umbilicus, skin, pleura, bladder, kidneys, pancreas, and even in males $[3,6]$.

However, the mechanism by which endometriosis is involved is still largely unknown. Significant effort has been undertaken to identify potential causes in the development of endometriosis, including retrograde menstruation, coelomic metaplasia, iatrogenic injury, and hematogenous or lymphatic dissemination [7]. Retrograde menstruation is believed to cause transcoelomic spread and implantation within the pelvis due to its predilection for gravity-dependent pelvic deposits in most observed lesions. However, this theory cannot explain distant and intraparenchymal lesions in atypical cases while the haematogenous or lymphatic dissemination may offer a better explanation for these atypical locations. The possible transportation of endometrial fragments is based on these vessels as vehicles in a fashion analogous to metastatic spread of neoplasm. The coelomic metaplasia may 

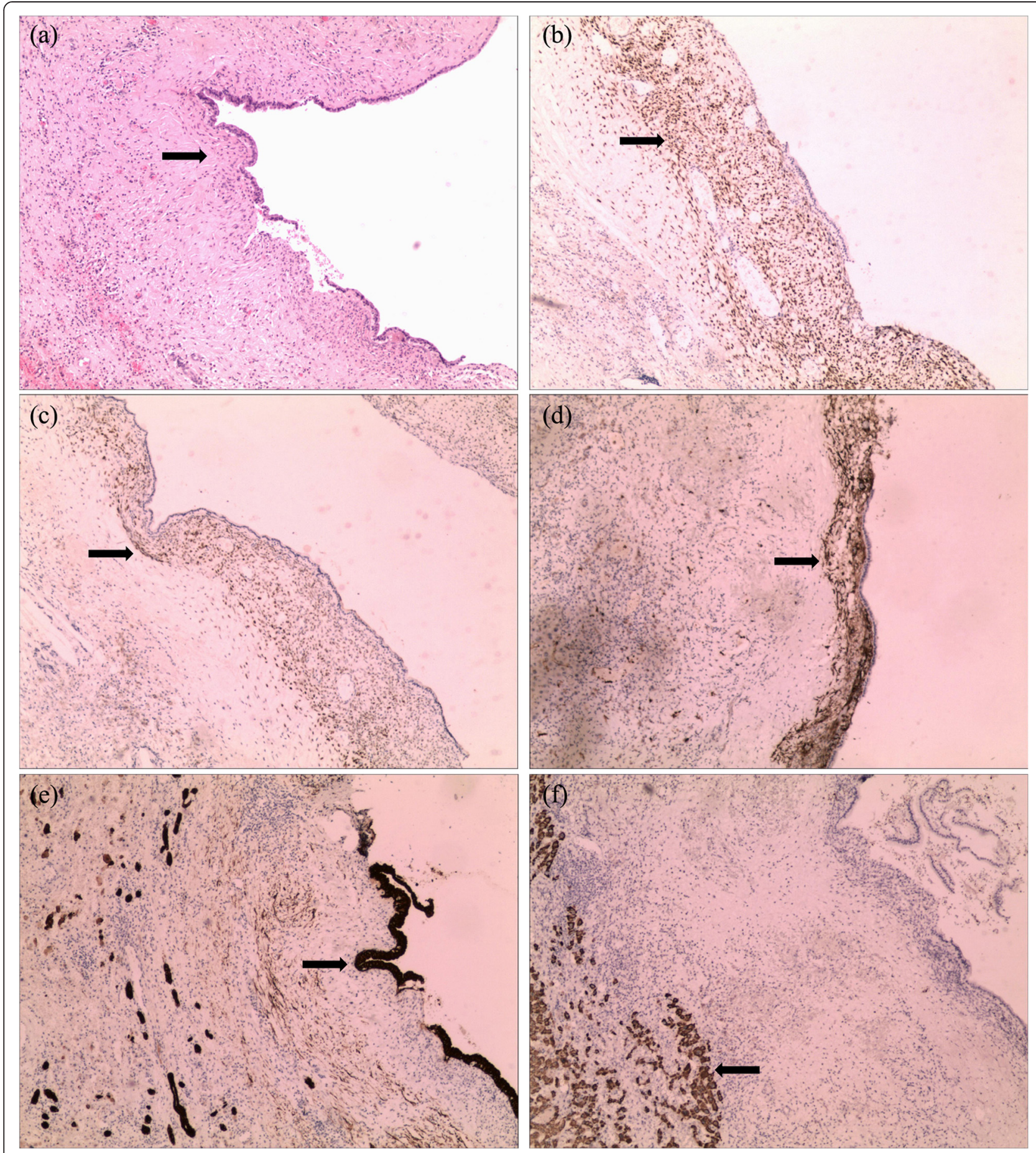

Figure 3 Microscopic examination of the endometriosis cyst of the liver. Cyst and normal liver tissue stained with hematoxylin and eosin (a) and with immunohistochemistry with estrogen receptor antibody (b), progesterone receptor antibody (c), CD10 antibody (d), CK7 antibody (e), and HepPar-1 antibody (f) (×100 magnification). No hyperplasia or atypia was observed in epithelial or stromal component.

be, at least in part, due to chronic inflammation, hormonal alternation, or trauma to promote endometriosis and that might be amenable to disruption in the male or heart $[6,8]$.
However, laboratory findings and clinical data of hepatic endometriosis fell short of metaplasia theory as most of the hepatic lesions are intraparenchymal. 
Hepatic endometriosis, first described by Finkel et al. in 1986, is an extremely rare disorder characterized by the presence of ectopic endometrial tissue in the liver [4]. The first reported patient was a 21-year-old female who presented with epigastric pain, nausea, and vomiting. The patient was found to have an endometrial cyst measuring $13 \mathrm{~cm}$ in diameter located in the left lobe of the liver. Subsequently, 21 cases of this rare form of endometriosis have been described in the medical literature. All cases of previously reported hepatic endometrioma and our present case are presented in Table 1. In our present case, the patient had no history of endometriosis or previous pelvic operation. By profiling the clinical and biological properties of the hepatic lesion, we may therefore identify vascular dissemination whose importance in hepatic intraparenchymal endometriosis was poorly recognized. We have now determined that in our case, the liver maybe a transportation target of endometrial fragments by lymphatic or blood vessels.

The clinical, biological, and morphological characteristics of hepatic endometriosis need to be reported in the literature to allow for the creation of a better diagnostic approach. These reported data suggest that, no specific diagnostic marker may be sufficient to isolate hepatic endometriosis from hepatic lesions except a histological examination, despite conducting a complete investigation, in the absence of clinical and radiological characteristics. Prospective identification of hepatic endometriosis has limitations that contribute to the controversy surrounding their existence. Although transhepatic biopsy for hepatic endometriosis have been sufficient to differentiate other hepatic lesions, it is difficult to make a preoperative diagnosis for the majority of hepatic endometriosis patients due to the potential adverse effects often available after transhepatic biopsy review. It is therefore enticing to suggest that systematic use of intraoperative frozen sections should be useful to avoid radical hepatectomy in order to decrease morbidity and mortality.

Hepatic endometrioma should always be considered in the differential diagnosis for a woman of any age presenting with a hepatic mass, with or without previous endometriosis history. The diagnosis is made on the basis of a histological examination, and a pericystectomy has been recommended.

\section{Conclusions}

In summary, our 36-year-old Chinese primiparous female with hepatic endometriosis forming a large cystic

Table 1 Feature of reported cases of hepatic endometriosis in literature

\begin{tabular}{|c|c|c|c|c|c|}
\hline Reference & Age (years) & Liver involvement & Endometrial history & Pre-operation diagnosis & Treatment \\
\hline Finkel et al. [4] & 21 & Left lobe & No & No & Cyst enucleation \\
\hline Grabb et al. [9] & 21 & Left lobe & No & No & Deroofing + danazol \\
\hline Rovati et al. [10] & 37 & Left lobe & Yes & Yes & Segmentectomy + danazol \\
\hline Verbeke et al. [1 1] & 62 & Left lobe & No & No & Excision \\
\hline Verbeke et al. [1 1] & 34 & Right lobe & No & No & Right hepatectomy \\
\hline Chung et al. [12] & 40 & Left lobe & Yes & No & Segmentectomy \\
\hline Weinfeld et al. [13] & 60 & Right lobe & Yes & No & Left hepatectomy + excision \\
\hline Inal et al. [14] & 25 & Right lobe & Yes & No & Danazol \\
\hline N'senda et al. [15] & 54 & Right lobe & No & Yes & Right hepatectomy \\
\hline Huang et al. [16] & 56 & Left lobe & Yes & No & Left hepatectomy \\
\hline Jeanes et al. [17] & 31 & Bilobar & Yes & Yes & Right hepatectomy \\
\hline Khan et al. [18] & 31 & Bilobar & Yes & Yes & Right hepatectomy + goserelin \\
\hline Khan et al. [18] & 59 & Right lobe & Yes & Yes & Right hepatectomy \\
\hline Tuech et al. [19] & 42 & Right lobe & No & No & Excision \\
\hline Reid et al. [20] & 46 & Right lobe & Yes & No & Right hepatectomy + goserelin \\
\hline Groves et al. [21] & 52 & Right lobe & No & No & Right hepatectomy \\
\hline Goldsmith et al. [22] & 48 & Left lobe & Yes & No & Non anatomical resection \\
\hline Asran et al. [23] & 61 & Bilobar & Yes & Yes & Unknown \\
\hline Schuld et al. [24] & 39 & Right lobe & No & No & Segmentectomy \\
\hline Fluegen et al. [25] & 32 & Right lobe & No & No & Pericystectomy \\
\hline Rivkine et al. [26] & 51 & Left lobe & No & No & Left hepatectomy \\
\hline Liu et al., this study & 36 & Left lobe & No & No & Pericystectomy \\
\hline
\end{tabular}


mass was diagnosed with endometriosis through histological immunostaining without intrinsic abnormality. Our present case suggested that the liver maybe a transportation target of endometrial fragments by lymphatic or blood vessels, which provides a theoretical basis for the complex mechanisms of endometriosis.

\section{Consent}

Written informed consent was obtained from the patient for publication of this case report and any accompanying images.

\section{Competing interests}

The authors declare that they have no conflict of interests.

\section{Authors' contributions}

$\mathrm{KL}$ and $\mathrm{YL}$ were responsible for the conception and design of the study. $\mathrm{KL}$, YW, FT and YT were responsible for the acquisition of data. KL, YW, and FT drafted the manuscript. All authors participated in interpretation of the findings. $K L$ and $Y L$ revised and commented on the draft, and all authors read and approved the final version of the manuscript. All authors confirm that the content has not been published elsewhere and does not overlap with or duplicate their previously published work.

\section{Acknowledgements}

This work was supported by grants from the Development and Reform Commission of Jilin Province (Grant No. 20130023-6).

\section{Author details}

'Department of Hepatobiliary and Pancreatic Surgery, No.71 Xinmin Street, First Hospital, Jilin University, Changchun 130021, China. ${ }^{2}$ Department of Thyroid Surgery, No.71 Xinmin Street, First Hospital, Jilin University, Changchun 130021, China.

Received: 22 October 2014 Accepted: 17 March 2015

Published online: 04 April 2015

\section{References}

1. Giudice LC. Clinical practice. Endometriosis. N Engl J Med. 2010;362:2389-98.

2. Haas D, Chvatal R, Reichert B, Renner S, Shebl O, Binder H, et al. Endometriosis: a premenopausal disease? Age pattern in 42,079 patients with endometriosis. Arch Gynecol Obstet. 2012;286:667-70.

3. Markham SM, Carpenter SE, Rock JA. Extrapelvic endometriosis. Obstet Gynecol Clin North Am. 1989;16:193-219.

4. Finkel L, Marchevsky A, Cohen B. Endometrial cyst of the liver. Am J Gastroenterol. 1986;81:576-8.

5. Johnson NP, Hummelshoj L, World Endometriosis Society Montpellier Consortium. Consensus on current management of endometriosis. Hum Reprod. 2013;28:1552-68.

6. Martin Jr JD, Hauck AE. Endometriosis in the male. Am Surg. 1985;51:426-30.

7. Agarwal N, Subramanian A. Endometriosis - morphology, clinical presentations and molecular pathology. J Lab Physicians. 2010;2:1-9.

8. Ceccaroni M, Clarizia R, Placci A. Pericardial, pleural, and diaphragmatic endometriosis. J Thorac Cardiovasc Surg. 2010;140:1189-90.

9. Grabb A, Carr L, Goodman JD, Mendelson DS, Cohen B, Finkel L. Hepatic endometrioma. J Clin Ultrasound. 1986;14:478-80.

10. Rovati V, Faleschini E, Vercellini P, Nervetti G, Tagliabue G, Benzi G. Endometrioma of the liver. Am J Obstet Gynecol. 1990;163:1490-2.

11. Verbeke C, Härle M, Sturm J. Cystic endometriosis of the upper abdominal organs. Report on three cases and review of the literature. Pathol Res Pract. 1996;192:300-4. discussion 305.

12. Chung CC, Liew CT, Hewitt PM, Leung KL, Lau WY. Endometriosis of the liver. Surgery. 1998:123:106-8

13. Weinfeld RM, Johnson SC, Lucas CE, Saksouk FA. CT diagnosis of perihepatic endometriosis complicated by malignant transformation. Abdom Imaging. 1998;23:183-4.
14. Inal M, Biçakçi K, Soyupak S, Oğuz M, Ozer C, Demirbaş O, et al. Hepatic endometrioma: a case report and review of the literature. Eur Radiol. 2000;10:431-4.

15. N'Senda P, Wendum D, Balladur P, Dahan H, Tubiana JM, Arrivé L. Adenosarcoma arising in hepatic endometriosis. Eur Radiol. 2000;10:1287-9.

16. Huang WT, Chen WJ, Chen CL, Cheng YF, Wang JH, Eng HL. Endometria cyst of the liver: a case report and review of the literature. J Clin Pathol. 2002;55:715-7.

17. Jeanes AC, Murray D, Davidson B, Hamilton M, Watkinson AF. Case report: hepatic and retro-peritoneal endometriosis presenting as obstructive jaundice with ascites: a case report and review of the literature. Clin Radiol. 2002;57:226-9.

18. Khan AW, Craig M, Jarmulowicz M, Davidson BR. Liver tumours due to endometriosis and endometrial stromal sarcoma. HPB (Oxford). 2002;4:43-5.

19. Tuech JJ, Rousselet MC, Boyer J, Descamps P, Arnaud JP, Ronceray J. Endometrial cyst of the liver: case report and review. Fertil Steril. 2003;79:1234-6.

20. Reid GD, Kowalski D, Cooper MJ, Kaloo P. Hepatic endometriosis: a case report and review of the literature. Aust N Z J Obstet Gynaecol. 2003;43:87-9.

21. Groves AM, Whitfield R, Lomas DJ, Gibbs P. Imaging of a hepatic endometrioma in a patient with multiple haemangiomas. J Hepatol. 2003;38:547.

22. Goldsmith PJ, Ahmad N, Dasgupta D, Campbell J, Guthrie JA, Lodge JP. Case hepatic endometriosis: a continuing diagnostic dilemma. HPB Surg 2009;2009:407206.

23. Asran M, Rashid A, Szklaruk J. Hepatic endometriosis mimicking metastatic disease: a case report and review of the literature. J Radiol Case Rep. 2010:4:26-31.

24. Schuld J, Justinger C, Wagner M, Bohle RM, Kollmar O, Schilling MK, et al. Bronchobiliary fistula: a rare complication of hepatic endometriosis. Fertil Steril. 2011;95:804.e 15-18.

25. Fluegen G, Jankowiak F, Zacarias Foehrding L, Kroepil F, Knoefel WT, Topp SA. Intrahepatic endometriosis as differential diagnosis: case report and literature review. World J Gastroenterol. 2013;19:4818-22.

26. Rivkine E, Jakubowicz D, Marciano L, Polliand C, Poncelet C, Ziol M, et al. Hepatic endometrioma: a case report and review of the literature: report of a case. Surg Today. 2013;43:1188-93.

\section{Submit your next manuscript to BioMed Central and take full advantage of:}

- Convenient online submission

- Thorough peer review

- No space constraints or color figure charges

- Immediate publication on acceptance

- Inclusion in PubMed, CAS, Scopus and Google Scholar

- Research which is freely available for redistribution 